\title{
Generalized Small Schröder numbers
}

\author{
JiSun Huh \\ Department of Mathematics \\ Yonsei University \\ Seoul, Republic of Korea \\ hyunyjia@yonsei.ac.kr
}

\author{
SeungKyung Park \\ Department of Mathematics \\ Yonsei University \\ Seoul, Republic of Korea \\ sparky@yonsei.ac.kr
}

Submitted: Nov 14, 2014; Accepted: Jul 13, 2015; Published: Jul 31, 2015

Mathematics Subject Classifications: 05A15

\begin{abstract}
We study generalized small Schröder paths in the sense of arbitrary sizes of steps. A generalized small Schröder path is a generalized lattice path from $(0,0)$ to $(2 n, 0)$ with the step set of $\{(k, k),(l,-l),(2 r, 0) \mid k, l, r \in \mathbf{P}\}$, where $\mathbf{P}$ is the set of positive integers, which never goes below the $x$-axis, and with no horizontal steps at level 0. We find a bijection between 5-colored Dyck paths and generalized small Schröder paths, proving that the number of generalized small Schröder paths is equal to $\sum_{k=1}^{n} N(n, k) 5^{n-k}$ for $n \geqslant 1$.
\end{abstract}

Keywords: generalized large and small Schröder paths; colored Dyck paths

\section{Introduction}

Lattice paths have been studied extensively by many mathematicians over a long period of time. Recently, several attempts of generalization have been successful. (See [2], [4], [7], and [3].) Schröder paths are also interesting objects, but have been rarely studied for any type of generalization until J.P.S Kung and A. De Mier [5] found the generating functions for the number of generalized Dyck and Schröder paths, which are called rook and queen paths, with a given right boundary and steps satisfying a natural slope condition. In this paper we try to enumerate combinatorially the number of the generalized small Schröder paths.

In the next section we give some basic definitions and facts on lattice paths in general. Section 3 talks about a bijection between colored Dyck paths and generalized small Schröder paths.

\section{Preliminaries}

We list two sets of definitions. 
Definition 1. The following are fairly well known, but we state them anyway.

(1) An $n$-Dyck path is a path from $(0,0)$ to $(2 n, 0)$ with up $U=(1,1)$ and down $D=(1,-1)$ steps that never goes below the $x$-axis.

(2) An $n$-Dyck path is called a prime if it never touches the $x$-axis except at the starting and terminal vertices.

(3) A peak in a Dyck path is a vertex between an up step and a down step, and a valley is a vertex between a down step and an up step.

(4) A double ascent in a Dyck path is an intermediate vertex of a consecutive $U U$ pair, and a double descent is defined similarly.

(5) The Narayana number $N(n, k)$ counts $n$-Dyck paths with $k$ peaks.

(6) An $n$-Schröder path is a path from $(0,0)$ to $(2 n, 0)$ with up $U=(1,1)$, down $D=(1,-1)$, and flat $F=(2,0)$ steps that never goes below the $x$-axis.

(7) An $n$-small Schröder path is a Schröder path having no flat steps at level 0.

Definition 2. We introduce the new ones that we need throughout this paper.

(1) A 5-colored $n$-Dyck path is an $n$-Dyck path only whose double ascents are colored in one of five colors, b(black),w(white), f(flame), g(green), and y(yellow).

(2) A bicolored $n$-small Schröder path is an $n$-small Schröder path whose vertices between distinct steps of $U, D$, and $F$ are colored in $b$ only and vertices between the same kind of steps of $U U, D D$, and $F F$ are colored in either $b$ or $w$. The starting vertex and ending vertex are not colored.

(3) A generalized (large) $n$-Schröder path is a lattice path from $(0,0)$ to $(2 n, 0)$ using steps from $\{(k, k),(l,-l),(2 r, 0)\}$ for any positive integers $k, l$, and $r$, that never goes below the $x$-axis.

(4) A generalized $n$-small Schröder path is a generalized $n$-Schröder path with no flat steps at level 0 .

Then the following lemma is straightforward from the definitions 2.1 (5) and 2.2 (1). (The number is listed as A078009 in OEIS [6].)

Lemma 3. The number of 5-colored $n$-Dyck paths is $\sum_{k=1}^{n} N(n, k) 5^{n-k}$. 


\section{A bijection between 5-colored Dyck paths and generalized small Schröder paths}

In this section we provide a bijective relation between 5 -colored $n$-Dyck paths and bicolored $n$-small Schröder paths by Bijection I. Then we give a natural bijection between bicolored $n$-small Schröder paths and generalized $n$-small Schröder paths by Bijection II. In other words, we prove the following theorem bijectively.

Theorem 4. The number of generalized n-small Schröder paths is

$$
\sum_{k=1}^{n} N(n, k) 5^{n-k} .
$$

\section{Proof. Bijection I.}

Let $P$ be a 5 -colored $n$-Dyck path. We construct the corresponding bicolored $n$-small Schröder paths by taking four steps described as follow:

STEP 1. (coloring)

For each double ascent of $P$ find the first double descent right of the ascent at the same level. We call this the matching double descent. Note that each double ascent of $P$ is colored in one of five colors, $b, w, f, g$, and $y$. Let $P^{\prime}$ be the path obtained from $P$ by the following process:

(i) If a double ascent of $P$ is colored in one of three colors, $b, w$, and $f$, then color its matching double descent in the same color.

(ii) If a double ascent of $P$ is colored in one of two colors, $g$ and $y$, then replace the color with $b$ and $w$, respectively, and color its matching double descent in $w$ and $b$, respectively. (iii) Color each peak and valley of $P$ in $b$.

Notice that every $f$ colored double ascent in $P^{\prime}$ has the matching double descent also colored in $f$.

STEP 2. (factorizing)

Find the first vertex $v$ colored in $f$. Assume that $v$ is the ending vertex of an up step $U$ at level $i$. Then one can find the corresponding first down step $D$ at the same level. Let $Q$ be the subpath of $P^{\prime}$ above the level $i$ from $U$ to $D$. Then we can factor $P^{\prime}$ in general as $L U f Q x D R$, where $L$ and $R$ are subpaths of $P^{\prime}, f$ is the color of the vertex $v$, and $x$ is a color of the starting vertex of $D$ which is either $b$ or $f$. Initially, this $x$ is $f$, but as processes repeated it can be $b$ if $Q$ ends with a flat step. (See Figure 1 (i).)

STEP 3. (creating flats)

In the subpath $U f Q x D, Q$ can be factored as $Q_{1} b Q_{2}$ where $Q_{1}$ is the first prime path at level $i$ and $Q_{2}$ is the rest of the path of $Q$. If $Q$ is a prime then take $Q_{1}=Q$. (See Figure 1 (ii).)

Now find the lowest level $k$ of $Q_{1}$ at which a black colored vertex lies. 


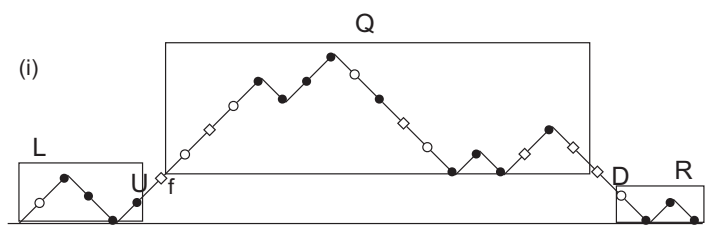

(ii)

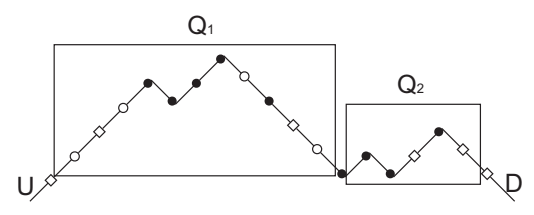

Figure 1: The factorizations of $P^{\prime}$, (i) and $Q$, (ii). A square vertex represents a vertex of color $f$.

Note that

(i) $Q_{1}$ starts with $k-i U$ steps and ends with $k-i D$ steps.

(ii) First $k-i-1$ double ascents are colored in $w$ or $f$

(iii) Each of the last $k-i-1$ double descents is colored in the same color of its matching double ascent.

Thus we can factor $Q_{1}$ as $Q_{1}=U_{1} c_{1} U_{2} \cdots c_{k-i-1} U_{k-i} c_{k-i} Q_{1}^{*} c_{k-i+1} D_{k-i} c_{k-i-1} \cdots D_{2} c_{1} D_{1}$, where $Q_{1}^{*}$ is a subpath of $Q_{1}$ above the level $k, U_{j}$ is the up step ending at the level $i+j$, and $D_{j}$ is the down step starting at the level $i+j$ for $j=1,2, \cdots, k-i$.

We have four cases to construct flat steps depending on the colors of vertices $c_{k-i}$ and $c_{k-i+1}$, i.e., the starting and ending vertices of the subpath $Q_{1}^{*}$, which are:

Case 1: The color $c_{k-i+1}$ is $b$.

Case 2: The colors of $c_{k-i}$ and $c_{k-i+1}$ are both $f$.

Case 3: The color $c_{k-i}$ is $b$ and $c_{k-i+1}$ is $w$.

Case 4: The colors of $c_{k-i}$ and $c_{k-i+1}$ are both $w$.

We explain these four cases one at a time, all of which will be concluded by the last process of changing colors. We start at the subpath $U f Q x D$ of the path $P^{\prime}$.

Case 1.

(i) Erase the color $f$ of $U f Q x D$ and delete $U_{1} c_{1} U_{2} \cdots c_{k-i-1} U_{k-i}$ of $Q_{1}$, so that the color $c_{k-i}$ takes over the color $f$.

(ii) Replace each down step $D_{j}(1 \leqslant j \leqslant k-i)$ of $Q_{1}$ by $F_{j}$ at level $i$ with colors unchanged.

(iii) Replace the $x$ of $U f Q x D$ by $b$ if it is an $f$. 
Then the path $U f Q x D$ is now transformed to

$$
U c_{k-i} Q_{1}^{*} c_{k-i+1} F_{k-i} c_{k-i-1} \cdots F_{2} c_{1} F_{1} b Q_{2} b D \text {. (See Figure 2.) }
$$

If the $Q_{1}^{*}$ is empty, then $c_{k-i}=c_{k-i+1}$ and then flat steps followed.

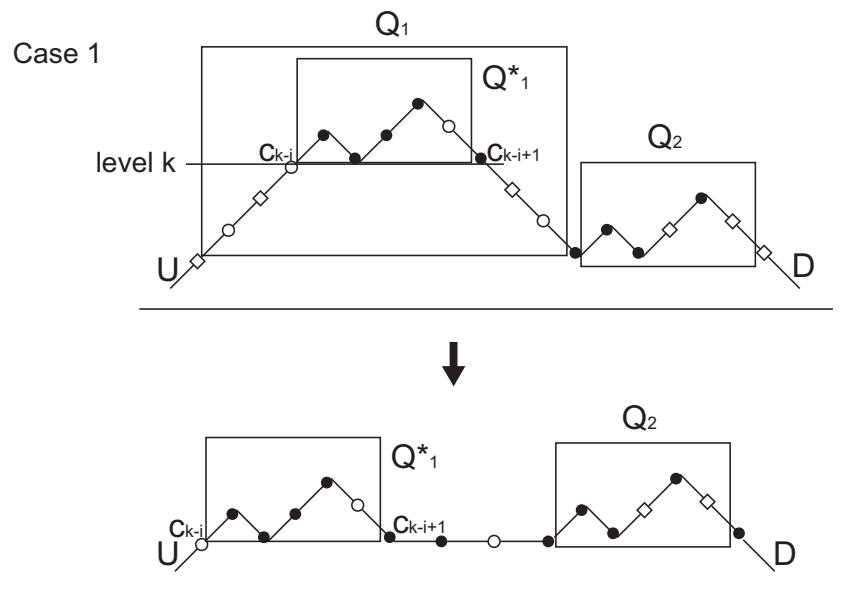

Figure 2: Case 1.

Case 2.

The first three processes are the same as Case 1. We just change the color of the first flat step from $c_{k-i+1}=f$ to $b$.

Then the path $U f Q x D$ is transformed to $U c_{k-i} Q_{1}^{*} b F_{k-i} c_{k-i-1} \cdots F_{2} c_{1} F_{1} b Q_{2} b D$. (See Figure 3.)

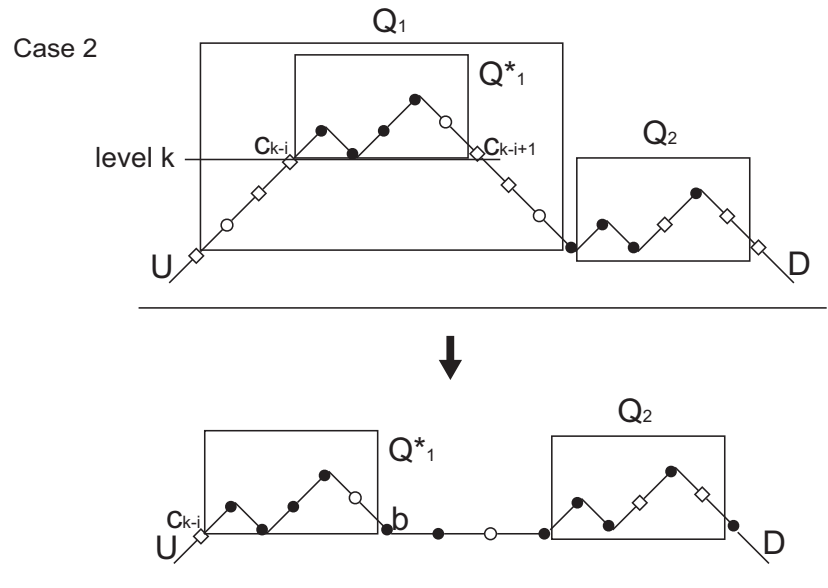

Figure 3: Case 2. 
Case 3.

(i) Replace the color $f$ of $U f Q x D$ with $b$ and erase the color $x$.

(ii) Replace each up step $U_{j}(1 \leqslant j \leqslant k-i)$ of $Q_{1}$ by $F_{j}$ at level $i$ with colors unchanged.

(iii) Delete $D_{k-i} c_{k-i-1} \cdots D_{2} c_{1} D_{1}$ of $Q_{1}$.

(iv) Cut off $b Q_{2}$ of $Q=Q_{1} b Q_{2}$ and then paste it after turning 180 degrees (denoted by $\bar{Q}_{2} b$ ) in between the first color $b$ in (i) and the first flat step $F_{1}$ in (ii). (See Figure 4 ).

Then the path $U f Q x D$ is transformed to $U b \bar{Q}_{2} b F_{1} c_{1} \cdots F_{k-i} c_{k-i} Q_{1}^{*} c_{k-i+1} D$.

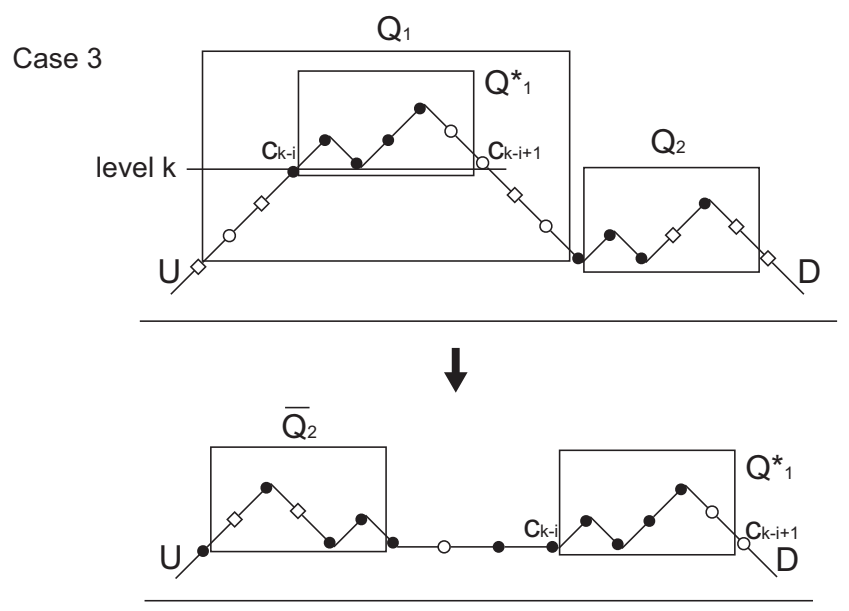

Figure 4: Case 3.

Case 4.

In this case, $Q_{1}^{*}$ is clearly neither empty nor prime. So we can factor $Q_{1}^{*}$ as $Q_{11}^{*} b Q_{12}^{*}$, where $Q_{11}^{*}$ is the first prime of $Q_{1}^{*}$ and $Q_{12}^{*}$ is the rest of $Q_{1}^{*}$.

(i) Erase the color $f$ of $U f Q x D$ and replace the $x$ with $b$.

(ii) Delete $U_{1} c_{1} U_{2} \cdots c_{k-i-1} U_{k-i}$.

(iii) Replace each down step $D_{j}(1 \leqslant j \leqslant k-i)$ of $Q_{1}$ by $F_{j}$ at level $i$ with colors unchanged.

(iv) Let $Q_{2}^{\prime}$ represent the newly created flat steps by (iii) along with $Q_{2} b$ from (i), and after turning 180 degrees of $Q_{2}^{\prime}$ let us denote it by $\bar{Q}_{2}^{\prime}$. Now cut off $Q_{2}^{\prime}$ and then paste $\bar{Q}_{2}^{\prime}$ after $Q_{11}^{*}$. (See Figure 5.)

Then the path $U f Q x D$ is transformed to $U c_{k-i} Q_{11}^{*} \bar{Q}_{2}^{\prime} b Q_{12}^{*} c_{k-i+1} D$. 


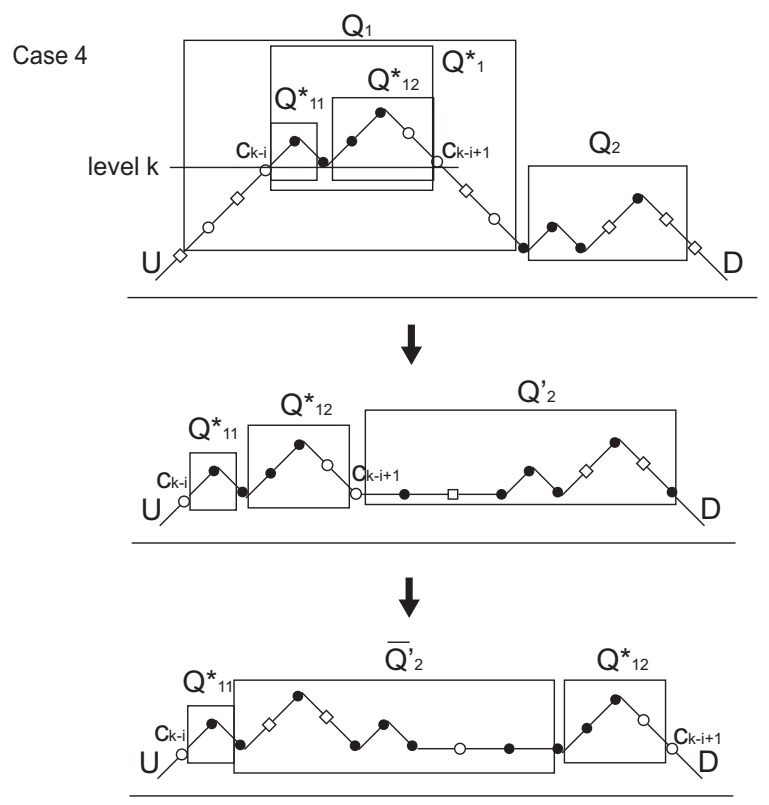

Figure 5: Case 4.

For the last process of Step 3 we replace the color $f$ 's between the flat steps with $b$ 's.

STEP 4. Repeat Step 2 and 3 until there is no vertex colored in $f$.

Then clearly the outcome is a bicolored $n$-small Schröder path.

Now we give an example, which can be obtained by applying STEPs 1 through 4 along with Figures 1 through 5.

Let a 5-colored 16-Dyck path $P$ be given:

$$
P=U f U f U w U f U f U f U f U w U D U D D U D D D U f U D^{5} U f f U y U D^{5},
$$

where $D^{5}$ represents $D D D D D$. We will construct the corresponding bicolored 16 -small Schröder path $\hat{P}$ :

$$
\hat{P}=U w U b F b U b F b U b D w D b F w F b F b U b F b D b F b F b U b D b F b U b D w D .
$$

Step by step applications of STEPs and Cases are showed in the Figure 6 .

For recovery processes we first note that from STEP 3: 


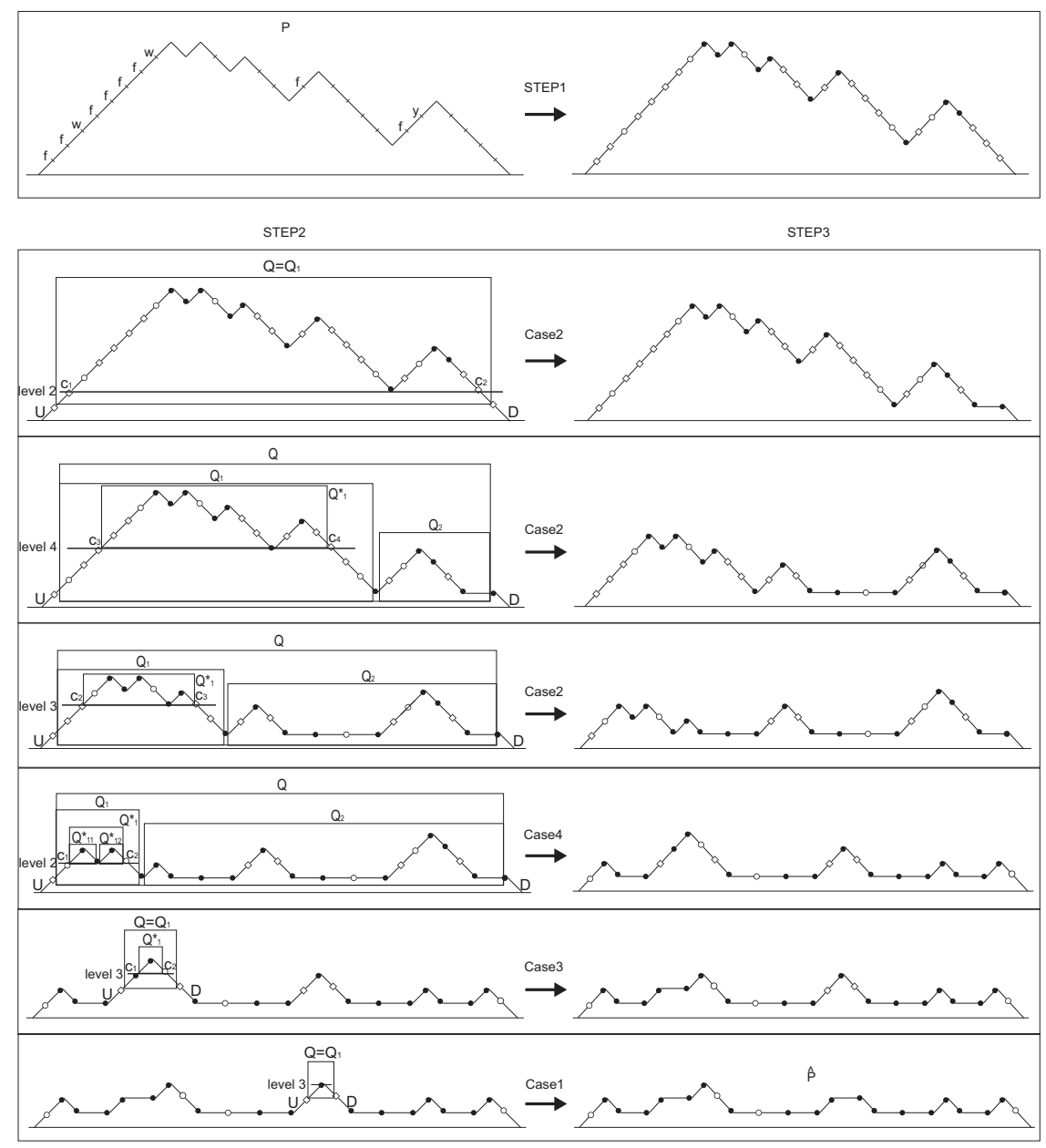

Figure 6: Example 3. Transforming $P$ to $\hat{P}$

1. The outcomes of Cases 1 and 2 give the color $b$ to the starting vertex of the $D$ in $U f Q x D$, and the leftmost block of F's on the level $i$ is created lastly.

2. Cases 3 and 4 produce the color $w$ to the starting vertex of the $D$ in $U f Q x D$, and the rightmost block of $F$ 's on the level $i$ is created lastly.

3. In any case, if $Q_{2}$ is not empty, then it should not start with a flat step.

Now we describe the recovery process. Let $\hat{P}$ be a bicolored $n$-small Schröder path. First, we label the $i$ th up step by $U_{i}$ from left to right. Let $U_{i}$ be an up step ending at the level $k$ and let $U_{j}(i<j)$ be the next up step ending at the level $k$. Then the flat steps at the level $k$ between these up steps $U_{i}$ and $U_{j}$ are labeled as $F_{i}$. If there is no such $U_{j}$, then every flat step at level $k$ is labeled as $F_{i}$. We call this $U_{i}$ a label up step for the flat steps. We also call the first down step after $U_{i}$ starting at the same level the label down step for the flat steps. 
Now find the flat steps whose subscript is the largest. These steps can be appeared as blocks of consecutive flat steps. Then look for the starting vertex $v_{2}$ of the label down step for these flat steps. Let $v_{1}$ be the ending vertex of the corresponding label up step, for convenience. Then we take the following processes to recover the corresponding Dyck path just before the STEP 1.

1. If $v_{2}$ has color $b$, then the leftmost block of flat steps are changed first. If the color of $v_{1}$ is $b$ or $w$, then we apply the reverse process of Case 1 of STEP 3 . If the color of $v_{1}$ is $f$, then apply the reverse process of Case 2 of STEP 3.

2. If $v_{2}$ has the color $w$, then the rightmost block of flat steps are changed first. If the color of $v_{1}$ is $b$, then we apply the reverse process of Case 3 of STEP 3 . If the color of $v_{1}$ is $w$, then apply the reverse process of Case 4 of STEP.

3. Repeat these processes until we have no flats.

Then we have an $n$-Dyck path whose double ascents and double descents are colored in one of three colors, $b, w$, and $f$, and all peaks and valleys are colored in $b$. By the following recovery processes of colors, we can fully recover the 5 -colored $n$-Dyck path $P$ :

1. Delete the colors of peaks and valleys.

2. If a double ascent and its matching double descent are in the same color, then delete the color of the double descent.

3. If not, give each double ascent a new color by the following:

(a) Color the double ascent in $g$ if a pair of the colors of a double ascent and its matching double descent is $(b, w)$ and then delete the color of the double descent.

(b) Color the double ascent in $y$ if a pair of the colors of a double ascent and its matching double descent is $(w, b)$ and then delete the color of the double descent.

Now we have the 5 -colored $n$-Dyck path as we desired. Figure 7 shows full recovery processes.

Let us consider the second bijection. As we mentioned earlier, this bijection gives oneto-one correspondence between bicolored $n$-small Schröder paths and generalized $n$-small Schröder paths.

\section{Bijection II.}

Let $\hat{P}$ be a given bicolored $n$-small Schröder path. Recall that in $\hat{P}$ all peaks and valleys are colored in black, and the starting and ending vertices of all blocks of flats are colored in black. And other vertices can be black within blocks of up, down, and flat 


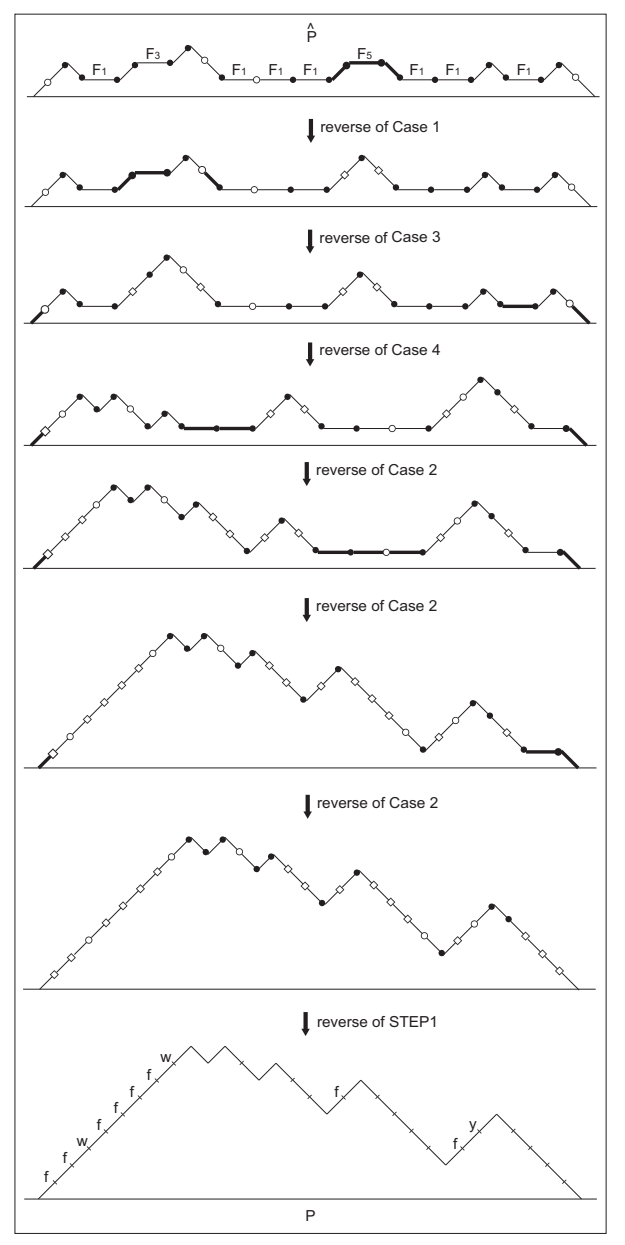

Figure 7: The recovery processes of Example 3

steps, respectively. So these black vertices separate the path $\hat{P}$ into line segments. By taking each of these line segments as a step we have the generalized $n$-small Schröder path, denoted by $\tilde{P}$, which has arbitrary lengths of steps. And the recovery process is pretty obvious. (See Figure 8.)
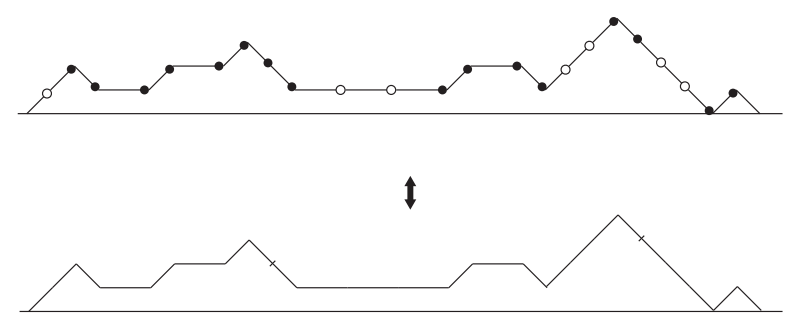

Figure 8: An example of Bijection II. 


\section{Acknowledgements}

Authors would like to thank an anonymous referee for his or her kind advice.

\section{References}

[1] P. Barry and A. Hennessy. A note on Narayana triangles and related polynomials, Riordan arrays, and MIMO capacity calculations. Integer Seq., 14, 2011.

[2] C. Coker. Enumerating a class of lattice paths. Disc. Math., 271: 13-28, 2003.

[3] J. Huh and S. Park. The Chung-Feller Theorem on Generalized Lattice Paths. Submitted.

[4] A. Huq. Generalized Chung-Feller Theorems for Lattices Paths. PhD Thesis. Brandeis University, 2009. arXiv:0907.3254.

[5] J.P.S Kung and A. De Mier. Catalan lattice paths with rook, bishop and spider steps. J. Combinatorial Theory Ser. A, 120(2):379-389, 2013.

[6] OEIS. The on-line encyclopedia of integer sequences. Available at: http://oeis. org/.

[7] J. Rukavicka. On generalized Dyck paths. Electron. J. Combin., 18: \#P40, 2011. 